\section{Towards A Computational Prediction of Nanoparticle Pharmacokinetics and Distribution}

\section{Abstract}

A number of nanotechnology-enabled drug delivery platforms are in varying stages of drug development. While, physiologically-based pharmacokinetic (PBPK) modelling has become a well-established tool for conventional medicines from preclinical to post-licensing environments, its application for nanomedicines is in its infancy. Part of the reason for this is that the fundamental mechanisms that underpin drug-handling within the body are not as well understood for nanoparticles. A number of recent initiatives aim to further develop PBPK modelling for nanomedicine applications but more fundamental knowledge of the relevant anatomical, physiological and pharmacological processes influencing distribution is required for robust computational prediction.

Received: January 07, 2016; Accepted: February 24, 2016; Published: February 29, 2016

\section{Purpose}

The purpose of this editorial is to summarise progress towards robust physiologically-based pharmacokinetic modelling approaches as applied to the development of nanomedicines. The authors stress the need for additional mechanistic data regarding the interaction between nanoparticles and biological systems, needed to inform such in silico approaches. Finally, a number of recently funded initiatives are overviewed that should help address some of the current knowledge gaps.

\section{Main Text}

The delivery of therapeutic agents is characterised by numerous challenges including poor absorption, low penetration into target tissues and non-specific dissemination throughout the body. Collectively, these issues can influence, drug exposure as well as toxicity and ultimate efficacy, and therefore robust prediction in early development is highly desirable. Several nanomedicine strategies have emerged as advanced approaches to enhance drug delivery and improve the pharmacodynamics across several diseases, although a particular emphasis has been placed on cancer due to early recognition of the enhanced permeation and retention effect. Nanoparticles for drug delivery can find application either by forming solid polymer matrix nanoformulations to encapsulate drugs, or through the construction of vehicles such as micelles, block copolymer Marco Siccardi ${ }^{1}$ and
Andrew Owen

\author{
1 Department of Molecular and Clinical \\ Pharmacology, Institute of Translational \\ Medicine, University of Liverpool, UK \\ 2 European Nanotechnology \\ Characterisation Laboratory, Department \\ of Molecular and Clinical Pharmacology, \\ University of Liverpool, UK
}

Corresponding author: Marco Siccardi

siccardi@liverpool.ac.uk

Department of Molecular and Clinical Pharmacology, Institute of Translational Medicine, University of Liverpool, 70 Pembroke Place, Liverpool, L69 3GF, UK.

\section{Tel: +44 (0) 1517948211}

Fax: + 44 (0) 1517945656

Citation: Siccardi M, Owen A. Towards A Computational Prediction of Nanoparticle Pharmacokinetics and Distribution. J In Silico In Vitro Pharmacol. 2016, 2:1.

liposomes / vesicles and nanoemulsions [1]. Different inorganic oxides such as such as gold, silver, silica and iron have been used to develop nanoparticles which than can be conjugated with therapeutic agents. The application of nanotechnology to drug delivery is gaining momentum and is expected to dramatically proliferate over the coming years. To date, 247 nanomedicine products have been approved or are in various stages of clinical study [2] and the global nanomedicine market was recently predicted to reach $\$ 528$ billion by 2019 [3]. In the last 10 years, it is estimated that more than one thousand companies have been engaged in development of nanotechnology applications in medicine (source: NanoWerk database). The increasing popularity of nanomedicines is also exemplified by the increase in the associated literature, with the number of articles associated with the search term "nanoparticle" now having comfortably exceeded that of "monoclonal antibody" (Figure 1).

The processes that underpin exposure to nanomedicines are not as well understood as conventional medicines and current early 
preclinical development places great emphasis on rigorous in vivo assessment. Since efficacy and toxicity of therapies can be influenced by nanoparticle distribution [4], the appropriateness of existing regulation for nanomedicines has been the subject of significant recent debate $[5,6]$. Consequently, understanding the interactions between nanomaterials and the human body is of great importance to efficient development of safe and effective new drug delivery systems, and requires a rigorous understanding of how the physical properties of the materials superimpose upon the relevant anatomy, physiology and pharmacology.

Physiologically-based pharmacokinetic (PBPK) modelling is now widely recognised as a powerful pharmacological tool to simulate the pharmacokinetics and distribution of drugs. PBPK modelling can be applied to medicine optimisation either during preclinical / clinical development or post-licensing. Indeed, the European Medicines Agency (EMA) and the US Food and Drug Administration (FDA) regulatory guidelines were recently updated to include PBPK modelling for the purpose of dose finding, assessing drugdrug interactions, and investigating the utility of dose adjustment in special populations (e.g. during pregnancy). PBPK modelling integrates in vitro drug specific data into a mathematical description of the anatomical, physiological and molecular processes mediating absorption, distribution, metabolism and elimination (ADME) to simulate pharmacokinetics. Importantly, the traction of PBPK modelling as an in silico tool for predicting pharmacokinetics in vivo in recent years has been underpinned by the robustness of the knowledge of processes important in determining small molecule ADME. In addition to the wide industrial and regulatory uptake of PBPK modelling, the approach is also being adopted in the academic environment. Accordingly, there are now over 1200 publications listed in Pubmed with the search term "PBPK" (Figure 1).

As well as simplifying the drug development process, by providing an estimation of the expected pharmacokinetics in humans or in pre-clinical species, PBPK modelling has the potential to dramatically reduce the number of animals needed during preclinical development [7]. Thus, validated nano-specific PBPK modelling approaches represent a pivotal advancement for refining, replacing and reducing the use of preclinical species during development programmes, as has been achieved for conventional medicines. However, modelling approaches for nanomedicine applications are currently in their infancy compared to small molecule drugs with fewer than 20 manuscripts listed in Pubmed for the search term "nanoparticle + PBPK" at the time of writing. The processes regulating pharmacokinetics and distribution of nanoparticles are known to differ from conventional small molecule drugs, and there is a current paucity of knowledge across different nanomedicine platform technologies, which are needed for the development of robust predictive tools.

It is clear that PBPK modelling can assist in answering questions that cannot otherwise be examined in pre-clinical development without a heavy burden on pre-clinical species. The approach provides the opportunity for rational design of nanomedicines, identifying strategies to maximise the efficacy and safety of

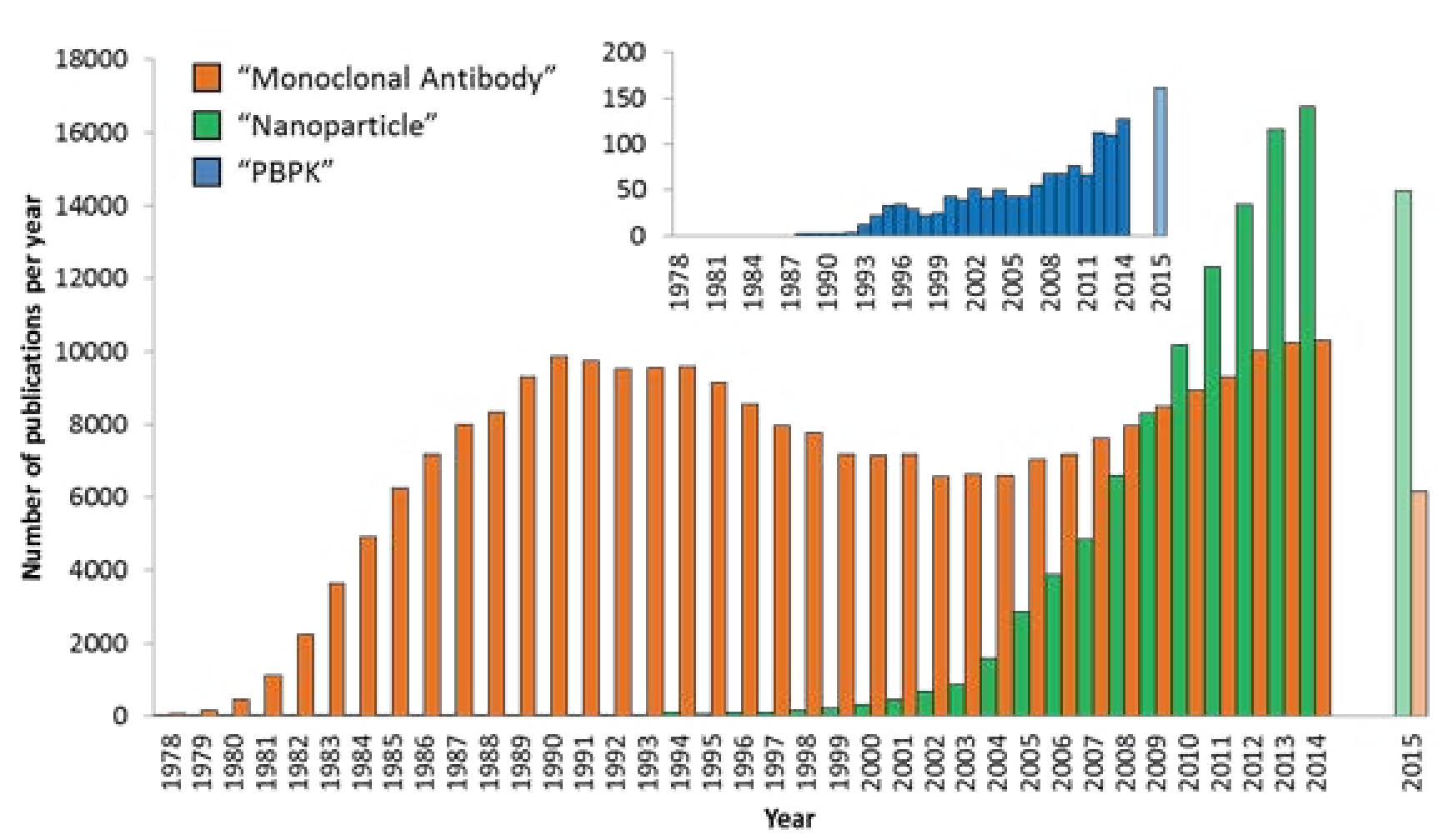

Figure 1 Publications per annum listed in PubMed between 1978 and 2015 for the search terms "monoclonal antibodies" versus "nanoparticles". The inset demonstrates the number of publications for "PBPK" over the same timeframe. 
novel technologies. However, a better mechanistic understanding of the molecular, anatomical and physiological events that define nanoparticle distribution is required, and will serve to have a beneficial impact on development of novel nanoparticle assessment strategies. The authors have been active in development of PBPK models for traditional drug distribution simulating clinical scenarios such as drug interactions, dose personalisation, adjustment for special populations [8-12], and have also recently started to develop models for predicting pharmacokinetics of nanomedicine candidates [13]. Recently, we described innovative PBPK models to inform the development of long-acting injectable nanoformulations for anti-HIV therapy, predicting the optimal dose and drug release rates across a panel of commonly prescribed drugs [14]. Additionally, we recently described a PBPK model for the predicting distribution of superparamagnetic iron oxide nanoparticles (SPIONs) in animals and humans, providing a quantitative estimate of nanoparticle diffusion and accumulation in tissues and organs [15]. However, more work is required to realise the potential of the approach for nanomedicines, and the authors are playing an active role in two initiatives that should help address some of the current gaps in knowledge. Firstly, the US National Institutes of Health (NIH) recently funded the Long Acting Extended Release Resource Program (LEAP) that has a PBPK modelling core at the heart of its ambition. The LEAP modelling core will be available as a resource to stake holders actively involved in the development of long-acting nanoformulations for infectious diseases. Secondly, the European Commission recently funded the European Nanotechnology Characterisation Laboratory (EU-NCL) to provide a trans-European infrastructure, providing materials characterisation to developers to help facilitate nanomedicine translation. The EU-NCL will address structure activity relationships (SAR) for nanomaterials that should address some of the knowledge gaps relating to nanoparticle-biological interactions.

\section{Conclusion}

Although there are clear barriers to achieving widespread uptake of PBPK modelling in the nanomedicine community, which are driven by model complexity and current gaps in knowledge, significant adoption is expected in the future due to the high priority already given by regulatory agencies to the approach.
Future research efforts should focus upon a critical understanding of the interactions between nanomaterials and relevant organs, with consideration of how these processes may differ according to physical properties of the nanomaterial including composition, size, surface charge and morphology.

\section{Competing Interests}

$\mathrm{AO}$ is co-inventor of patents relating to the development of nanoformulations for drug delivery applications and he has received research funding from Merck, Pfizer and AstraZeneca, consultancy from Merck and Norgine. MS has received research funding from ViiV and Janssen.

\section{Authors' Contribution}

Both authors contributed equally to the work in terms of concepts and writing of the manuscript.

\section{Authors' Information}

AO is Professor in the Department of Molecular and Clinical Pharmacology at the University of Liverpool and the current Chair of the British Society for Nanomedicine. His research focuses on development of novel nanomedicines to overcome poor and variable drug exposure, and to understand the mechanistic basis for variability in pharmacokinetics and pharmacodynamics.

MS is a Lecturer in the Department of Molecular and Clinical Pharmacology at the University of Liverpool. His research interests cut across pharmacokinetics and pharmacodynamics of novel nanomedicine and traditional formulations and he is particularly interested in the development of in silico models to simulate pharmacokinetics and relevant clinical scenarios in virtual patients.

\section{Acknowledgement}

The authors would like to thank the National Institutes for Health (R24 Al118397-01; R01Al114405), European Commission (654190) and Engineering and Physical Sciences Research Council (EP/G066272/1; EP/I038721/1; EP/K002201/1) for research funding. 


\section{References}

1 Wischke C, Schwendeman SP (2008) Principles of encapsulating hydrophobic drugs in PLA / PLGA microparticles. Int J Pharm 364: 298-327.

2 Etheridge ML, Campbell SA, Erdman AG, Haynes CL, Wolf SM, et al. (2013) The big picture on nanomedicine: the state of investigational and approved nanomedicine products. Nanomedicine 9: 1-14.

3 BCC Research (2015) Nanotechnology in Medical Applications: The Global Market.

4 Moss DM, Siccardi M (2014) Optimizing nanomedicine pharmacokinetics using physiologically based pharmacokinetics modelling. British journal of pharmacology 171: 3963-3979.

5 Bawa R (2011) Regulating nanomedicine - can the FDA handle it. Current drug delivery 8: 227-234.

6 Eifler AC, Thaxton CS (2011) Nanoparticle therapeutics: FDA approval, clinical trials, regulatory pathways, and case study. Methods in molecular biology 726: 325-338.

7 Thomas S (2009) Physiologically-based simulation modelling for the reduction of animal use in the discovery of novel pharmaceuticals. Alternatives to laboratory animals: ATLA 37: 497-511.

8 De Roche M, Siccardi M, Stoeckle M, Livio F, Back D, et al. (2012) Efavirenz in an obese HIV- infected patient--a report and an in vitroin vivo extrapolation model indicate risk of underdosing. Antiviral therapy 17: 1381-1384.

9 Siccardi M, Almond L, Schipani A, Csajka C, Marzolini C, et al. (2012) Pharmacokinetic and Pharmacodynamic Analysis of Efavirenz
Dose Reduction Using an In Vitro-In Vivo Extrapolation Model. Clin Pharmacol Ther 92: 494-502.

10 Moss DM, Siccardi M, Back DJ, Owen A (2013) Predicting intestinal absorption of raltegravir using a population-based ADME simulation. J Antimicrob Chemother.

11 Siccardi M, Marzolini C, Seden K, Almond L, Kirov A, et al. (2013) Prediction of drug-drug interactions between various antidepressants and efavirenz or boosted protease inhibitors using a physiologically based pharmacokinetic modelling approach. Clinical pharmacokinetics 52: 583-592.

12 Siccardi M, Olagunju A, Seden K, Ebrahimjee F, Rannard S, et al. (2013) Use of a physiologically-based pharmacokinetic model to simulate artemether dose adjustment for overcoming the drug-drug interaction with efavirenz. In Silico Pharmacology.

13 McDonald TO, Giardiello M, Martin P, Siccardi M, Liptrott NJ, et al. (2013) Antiretroviral Solid Drug Nanoparticles with Enhanced Oral Bioavailability: Production, Characterization, and In Vitro-In Vivo Correlation. Adv Healthc Mater.

14 Rajoli RK, Back DJ, Rannard S, Freel Meyers CL, Flexner C, Owen A, et al. (2015) Physiologically Based Pharmacokinetic Modelling to Inform Development of Intramuscular Long-Acting Nanoformulations for HIV. Clinical pharmacokinetics 54: 639-650.

15 Silva HA, Lima Jr E, Mansilla MV, Zysler RD, Pisciotti MLM, et al. (2015) Develoment of a physiologically based pharmacokinetic model to predict the superparamagnetic iron oxide nanoparticles (SPIONs) accumulation in vivo. In: 10th International Congress of Pharmaceutical Sciences - CIFARP Sao Paulo, Brasil. 